\title{
Ab Initio Study of the Edge States of Graphene Nanoribbons in the Presence of Electrodes
}

\author{
M. ZWIERZYCKI AND S. KROMPIEWSKI \\ Institute of Molecular Physics, Polish Academy of Sciences, M. Smoluchowskiego 17, 60-179 Poznań, Poland

\begin{abstract}
Thanks to its outstanding electronic properties, like very high mobility of carriers, graphene has emerged in recent years as exciting candidate for use in new electronic devices. When it is patterned in the form of ribbons with widths in the range of nanometers, its transport properties become strongly influenced by the presence of the states localized at the edges of the ribbon. Using first principles calculations we study the properties of these
\end{abstract} \\ states for both isolated ribbons and in the presence of metallic electrodes. The calculations were performed for \\ end contacted geometry i.e. for graphene sheets at right angle to the electrodes. Both para- and ferromagnetic \\ electrodes were considered.
}

PACS numbers: 73.20.At, 73.40.Ns

\section{Introduction}

The discovery of graphene, two-dimensional (2D) carbon allotrope, in 2004 [1] arose high hopes for its application in future electronic devices. The reason for this is high (exceeding $10^{5} \mathrm{~cm}^{2} /(\mathrm{Vs})$ ) mobility of carriers whose density and character (electrons or holes) can be controlled either by electric or chemical doping. The remarkable transport properties of graphene are related to peculiarity of its electronic structure, namely the fact that low-energy excitations can be described as massless, chiral Dirac fermions albeit with the speed of light replaced by 300 times lower Fermi velocity [2]. This leads to a range of interesting phenomena like finite minimal conductivity, the Klein paradox or the anomalous quantum Hall effect which can be observed even at room temperatures (see [3] for recent review). Additionally a successful spin-injection and spin coherence lengths exceeding $1000 \mathrm{~nm}$ have been demonstrated in graphene making it a promising candidate also for spintronic applications [4]. In the same context, it has been predicted that an interface between graphene and nickel or cobalt acts as perfect spin-filter [5].

When graphene is patterned in the form of a nanoribbon with zigzag edges, its properties can be strongly influenced by the formation of the localized edge states $[6,7]$. These turn out to be spin-polarized $[8]$ and interacting weakly across the ribbon with a tendency towards antiferromagnetic alignment of the magnetic moments [9]. It has been predicted that by controlling a magnetic structure along the edges (introducing or removing the domain walls) one can generate a substantial magnetoresistive effect [10].

The nature and properties of the edge states of isolated graphene ribbons seems to be well understood at present. Not much is known however about what happens when the ribbons and their edges are in contact with metallic electrodes necessary for transport measurements. In this contribution we present an $a b$ initio study of systems consisting of thin graphene nanoribbon with zigzag edge and infinite length sandwiched between metallic electrodes.
We choose geometry in which graphene is at the right angle to the surface of the electrodes, i.e. end-contacted geometry [11]. Such geometry is believed to be acceptable, especially for calculations of transport properties, for structures with highly transparent contacts. Experimental evidence in support of this view exists for related carbon nanotube structures [12].

The calculations were performed using the local spin density approximation (LSDA) of density functional theory (DFT). The specific approach used was full potential linearized augmented plane wave (FLAPW) method [13] as implemented in WIEN2k code [14]. The $\boldsymbol{k}$-point mesh density and the reciprocal lattice vector cut-off were chosen so as to ensure the charge convergence of $10^{-3}$ electrons per atom or less. The $\mathrm{C}-\mathrm{C}$ bond length was kept rigidly at $1.42 \AA$, identical to bulk graphite. The width of the ribbons was set to 12 carbon atoms from edge to edge. We did not optimize the geometry of the ribbon as the effect of the relaxation on electronic structure was demonstrated elsewhere to be negligible [15]. The systems with electrodes were modelled using supercells containing graphene ribbon and 4 monolayers (ML) of fcc(111) metal. The metal and graphene were assumed to be perfectly lattice matched, a reasonable approximation for $\mathrm{Cu}$ and $\mathrm{Ni}$, but less so for $\mathrm{Pd}$ (10\% mismatch). The results for the latter are therefore more qualitative than for the first two. The metal-graphene separation was calculated from the simple model of hard spheres which yields $1.32 \AA$.

\section{Results and discussion}

The results of calculations are summarized in Fig. 1. Turning first to isolated ribbons we compare the situation where the dangling bonds at the edges are left unsaturated with rather more realistic case of hydrogenated bonds. The relevant band structures and local densities of states for edge atoms are shown in parts (a) and (b), respectively, for the case of parallel alignment of edge moments. It turns out that when the bonds are left dangling both the in plane $\sigma\left(s p^{2}: s, p_{x}, p_{y}\right)$ and out of plane $\pi\left(p_{z}\right)$ 
orbitals give rise to spin-polarized edge states. The relevant states are identified by arrows in the figure. In this case the total magnetic moment per unit cell is equal to $2.5 \mu_{\mathrm{B}}$ with the polarization concentrated on the edges.
The local magnetic moments, defined by integral over spheres with radius equal to half the bond length, for the edge atoms reach $0.5 \mu_{\mathrm{B}}$.
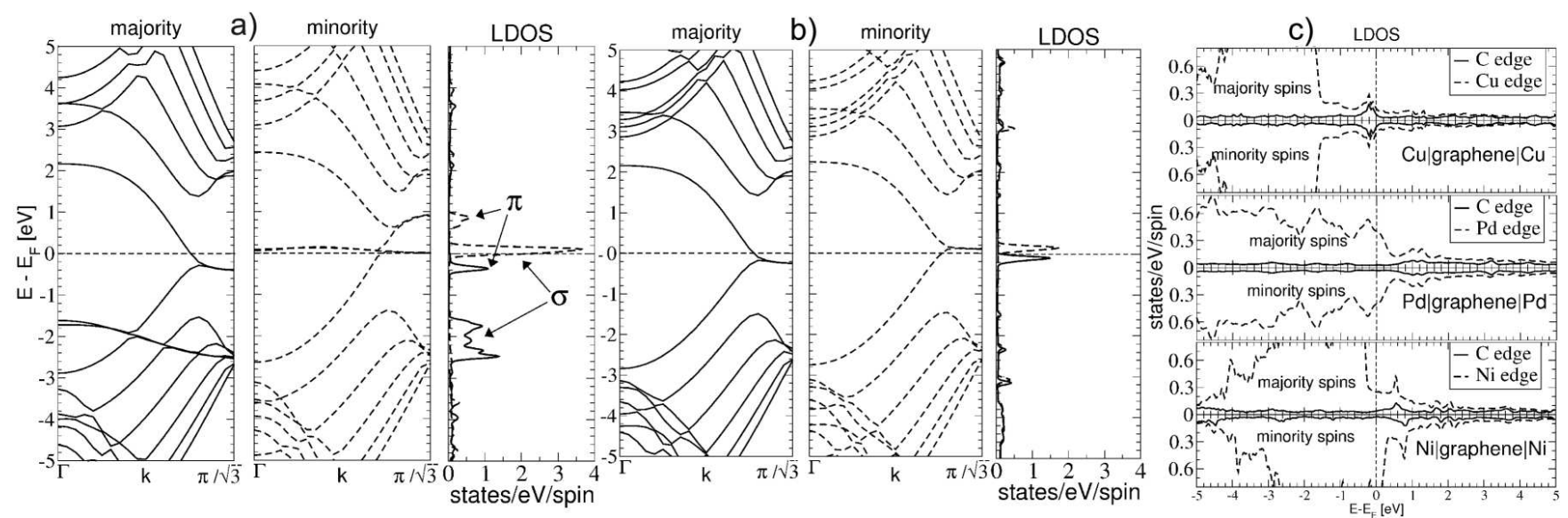

Fig. 1. Band structures and DOS of the edge atoms for nanoribbons with dangling (a) and hydrogenated (b) bonds;

(c) local DOS for interface atoms at graphene-metal interface.

Closer examination reveals that the main contribution to the magnetization comes from the dangling $\sigma$ bonds. In bulk graphene these are far removed from the Fermi level by bonding-antibonding splitting and can be usually neglected when discussing low lying excitations. When the bonds are saturated by hydrogen atoms $(\mathrm{C}-\mathrm{H}$ bond length was set to $1.08 \AA[9])$ the situation simplifies and the calculated band structure (part (b)) now exhibits only $\pi$-derived surface states in the vicinity of the Fermi level, a situation known from model calculations [6]. Total and local magnetic moments are reduced to 0.44 and $0.1 \mu_{\mathrm{B}}$, respectively.

Turning now to the system with electrodes (part (c)) we immediately see that the sharp features of local density of states (LDOS) seen for isolated ribbons are to a great extent washed away as a result of increased coordination (notice the difference of scales in LDOS plots between parts (a), (b) and (c)). Nonetheless some remaining features are visible just below the Fermi level for $\mathrm{Cu}$ |graphene interface giving rise to small local moments of less than $0.01 \mu_{\mathrm{B}}$. Surprisingly no magnetization whatsoever was found for pre-ferromagnetic Pd electrode. Although the double peaks visible in local DOS about $1 \mathrm{eV}$ above the Fermi level suggest the presence of the localized interface states (the peaks are absent in LDOS for central atoms), these are obviously not occupied and cannot give rise to ferromagnetic instability. As the last case we consider a system with magnetic $\mathrm{Ni}$ electrodes. Here the spin polarized but unoccupied edge states seem to be located around $0.8 \mathrm{eV}$ above the Fermi level. We do see some induced moments on the edge carbon atoms equal to about $0.01 \mu_{\mathrm{B}}$ and oriented opposite to the moment of the electrode. On Ni side there is a greatly reduced mag- netic moments $\left(0.1 \mu_{\mathrm{B}}\right.$ as compared to $0.6 \mu_{\mathrm{B}}$ for central layers) on the atoms in direct contact with graphene.

\section{Conclusion}

We have performed electronic structure calculations for graphene nanoribbons with and without metallic electrodes. We have found that while the edge states and related magnetic moments are generally reduced in the presence of electrodes, the details depend sensitively on the materials considered. For the case of ferromagnetic nickel the edge moments on graphene are oriented in the direction opposite to that of the electrode.

\section{Acknowledgments}

This work was supported by the Polish Ministry of Science and Higher Education as a research project No. N N202 199239.

\section{References}

[1] K.S. Novoselov, A.K. Geim, S.V. Morozov, D. Jiang, Y. Zhang, S.V. Dubonos, I.V. Grigorieva, A.A. Firsov, Science 306, 666 (2004).

[2] K.S. Novoselov, A.K. Geim, S.V. Morozov, D. Jiang, M.I. Katsnelson, I.V. Grigorieva, S.V. Dubonos, A.A. Firsov, Nature 438, 197 (2005).

[3] A.H. Castro Neto, F. Guinea, N.M.R. Peres, K.S. Novoselov, A.K. Geim, Rev. Mod. Phys. 81, 109 (2009).

[4] N. Tombros, C. Jozsa, M. Popinciuc, H.T. Jonkman, B.J. van Wees, Nature 448, 571 (2007).

[5] V.M. Karpan, G. Giovannetti, P. Khomyakov, M. Talanana, A. Starikov, M. Zwierzycki, J. van den Brink, G. Brocks, P.J. Kelly, Phys. Rev. Lett. 99, 176602 (2007).

[6] K. Nakada, M. Fujita, G. Dresselhaus, M. Dresselhaus, Phys. Rev. B 24, 17954 (1996). 
[7] Y. Kobayashi, K. Fukui, T. Enoki, K. Kusakabe, Phys. Rev. B 73, 125415 (2006).

[8] S. Okada, A. Oshiyama, Phys. Rev. Lett. 87, 146803 (2001).

[9] L. Pisani, J.A. Chan, B. Montanari, N.M. Harrison, Phys. Rev. B 75, 64418 (2007).

[10] W.Y. Kim, K.S. Kim, Nature Nano. 3, 408 (2008).

[11] S. Krompiewski, Phys. Rev. B 80, 075433 (2009).
[12] X. Song, X. Han, Q. Fu, J. Xu, N. Wang, D. Yu, Nanotechnology 20, 195202 (2009).

[13] D.J. Singh, Planewaves, Pseudopotentials and the LAPW Method, Kluwer, Boston 1994.

[14] K. Schwarz, P. Blaha, G.K.H. Madsen, Comp. Phys. Commun. 147, 71 (2002).

[15] H. Lee, Y. Son, N. Park, S. Han, J. Yu, Phys. Rev. B 72, 174431 (2005). 